\title{
Early experience using parallel grafting techniques for visceral artery preservation in the treatment of complex aortic aneurysms
}

\author{
Nathan W Kugler ${ }^{1}$, Rishi Subbaryan ${ }^{1}$, PeterJ Rossi ${ }^{1}$, ParagJ Patel ${ }^{2}$, Robert A Hieb ${ }^{2}$, Kellie R Brown ${ }^{1}$, Brian D Lewis ${ }^{1}$, Gary R Seabrook ${ }^{1}$ and \\ CheongJ. Lee ${ }^{*}$ \\ ${ }^{1}$ Division of Vascular Surgery, Department of Surgery, Medical College of Wisconsin, USA \\ ${ }^{2}$ Division of Interventional Radiology, Department of Radiology, Medical College of Wisconsin, USA
}

\begin{abstract}
Objective: Use of parallel grafting technique for visceral artery preservation is an alternative to prefabricated branched or fenestrated endovascular aortic repair (EVAR) for the treatment of paravisceral and thoracoabdominal aortic aneurysms. We report our early institutional outcomes following the use of "Snorkel" (SnEVAR) and "Sandwich" (San-EVAR) configurations to treat complex aortic aneurysms.

Methods: An IRB approved institutional review of the first 13 Sn-EVAR and San-EVAR cases at an academic medical center was performed and immediate perioperative outcomes were examined and analyzed. FDA approved and commercially available aortic endografts and peripheral devices were deployed in all cases. All devices were either delivered via an externalized axillary conduit or femoral artery access.

Results: Thirteen consecutive patients were treated using Sn-EVAR or San-EVAR techniques; seven males and six females with mean age of 71.9 years (SD 8.3 years). Eight patients had juxtarenal or pararenal aneurysms requiring primary Sn-EVAR and five had thoracoabdominal aneurysms where San-EVAR technique was used. Three patients had symptomatic aneurysms, one had an inflammatory aneurysm, and one patient had ruptured pathology prior to their repair. The mean aneurysm size was $60 \mathrm{~mm}$ (IQR 57-61 mm). Of the preoperatively planned 30 visceral vessel targets, 29 were successfully endografted and preserved (96.7\%). No individuals exhibited a type I endoleak, three patients (23.1\%) demonstrated type II endoleak, and a single (7.7\%) type III endoleaks occurred. Perioperative complications included one MI (7.7\%), one ascending aortic dissection with associated stroke (7.7\%), one complete T8 paralysis (7.7\%), single renal artery dissection (7.7\%), and one respiratory failure requiring tracheostomy (7.7\%). A single individual (7.7\%) required reintervention for retroperitoneal hemorrhage. The 30-day mortality rate was $7.7 \%$ with a single death on POD 4 after succumbing to complications of ascending aortic arch dissection noted on POD 1.
\end{abstract}

Conclusions: Initial experience with Sn-EVAR and San-EVAR demonstrates the technique to be a viable "off-the-shelf" alternative for treating complex aneurysms with acceptable perioperative outcomes.

\section{Introduction}

Endovascular repair of abdominal aortic aneurysms (EVAR) has become the standard of care for those with suitable anatomy since its inception in 1991 [1]. In 2005 the EVAR-1 trial was the first major study to demonstrate a 30-day mortality benefit when comparing EVAR to open aortic repair [2]. Subsequently in 2010 the DREAM study confirmed the findings of EVAR-1 while also demonstrating a decreased rate of 30-day major complications [3]. While EVAR has been proven to be a feasible method of repair, $30-40 \%$ of individuals remain excluded from EVAR repair due to unsuitable neck anatomy [4-7]. Fenestrated grafts (F-EVAR) were developed as a patient specific means for endovascular repair of patients with renal and/or visceral artery involvement [8]. There are limitations to F-EVAR in that an offthe-shelf system is not yet available with few still under investigative trials. Endograft construction time is required for F-EVAR and urgent and emergent applications are reserved to select physicians who are allowed to construct fenestrations on-table under an FDA approved Investigator Device Exemption.

Another approach to complex aortic neck anatomy, endovascular parallel grafting, was first described by Greenberg et al. in 2003 as a complementary technique to maintain visceral perfusion and achieve seal in the setting of a juxtarenal aneurysm [9]. Over the ensuing years this technique has continued to evolve and the spectrum of parallel grafting has expanded to include Snorkel (Sn-EVAR) and Sandwich (San-EVAR) configurations for the treatment of complex aortic aneurysms. We report our early institutional outcomes following the use of Sn-EVAR and San-EVAR techniques for treatment of complex juxtarenal, paravisceral, and thoracoabdominal aortic aneurysms.

\section{Methods}

An IRB approved institutional review of the first 13 planned Sn-EVAR and San-EVAR cases at an academic medical center was performed. Thirty-day perioperative outcomes were analyzed. FDA approved and commercially available aortic endografts and peripheral devices were employed in all cases. All devices were delivered either via

Correspondence to: Cheong J. Lee, MD, 9200 W Wisconsin Ave, Milwaukee, WI 53226, USA, Tel: 414-805-9160, Fax: 414-805-9170; E-mail: cjlee@mcw.edu

Received: September 15, 2015; Accepted: October 16, 2015; Published: October 20, 2015 
femoral access or via creation of an externalized axillary conduit.

\section{Imaging evaluation}

Pre-operative imaging included thoracic and abdominal computed tomography angiography (CTA). This was utilized to define the extent of aneurysm and all visceral vessel involvement while also assessing subclavian and aortic arch anatomy for preoperative planning.

Post-procedural imaging included a follow up CTA evaluation within one month along with plans for six month follow-up and yearly thereafter. Patients who had renal insufficiency and were unable to undergo CTA evaluation had duplex evaluation of visceral vessels to assess patency coupled with non-contract CT imaging for graft and aneurysm sac assessment.

\section{Procedural technique}

Procedural technique and overall steps have been previously well described [10-15]. Our technique varies slightly. All thoracoabdominal aortic aneurysms underwent Spinal Fluid Drain (SFD) placement the day prior to operative repair in order to allow pressure monitoring and drainage as necessary. Standard preoperative antibiotics were administered according to SCIP protocol and appropriately re-dosed throughout the case in accordance with pharmacy protocols. All patients were prepped chin to knees and draped to allow bilateral groin and axillary artery access. Systemic heparinization was achieved at the time of axillary and femoral artery access with Activated Clotting Times (ACT) maintained greater than 250 seconds for the remainder of the case.

An axillary cut down was performed on either the right or left axillary artery depending on preoperative imaging and planning. The axillary artery was isolated and a $10 \mathrm{~mm}$ PTFE graft was sewn in endto-side fashion to the artery providing an externalized conduit for antegrade delivery of endovascular devices. Bilateral femoral artery access was achieved utilizing either a total percutaneous "pre-close" technique or standard femoral cut-down methods as deemed necessary. Only commercially available "off-the-shelf" devices were utilized for the aortic reconstruction. All visceral arteries were preserved utilizing Viabahn (W. L. Gore and Assoc, Flagstaff, Ariz) self-expanding covered stents. Care was taken to ensure adequate overlap of the proximal segment of the Viabahn stent with the proximal margin of the aortic main body.

Adequate seal for primary Sn-EVAR repair was possible for patients with a juxtarenal aneurysm with a suprarenal aortic segment of $>15$ mm below the superior mesenteric artery. A San-EVAR was performed in cases of paravisceral and thoracoabdominal aortic morphology. All patients were started and maintained on dual antiplatelet therapy (aspirin and clopidogrel), if not on preoperatively,

\section{Results}

Thirteen consecutive patients were treated using Sn-EVAR or SanEVAR techniques; seven males and six females with mean age of 71.9 years (SD 8.3 years). Patient demographics including co-morbidities are outlined in Table 1 . Four patients $(31 \%)$ had undergone prior aortic aneurysm repair: two prior open repairs and two prior EVAR repairs. Eight patients had juxtarenal or paravisceral aneurysms requiring primary Sn-EVAR while five patients had thoracoabdominal aneurysms requiring San-EVAR repair. Three patients had symptomatic aneurysms, one patient had an inflammatory aneurysm, and one patient had ruptured pathology prior to repair. The median aneurysm
Table 1. Patient Demographics.

\begin{tabular}{|l|c|}
\hline Variable & Value \\
\hline $\begin{array}{l}\text { Age, mean } \pm \text { SD } \\
\text { (range) years }\end{array}$ & $71.9 \pm 8.3$ \\
\hline Gender, No. (\%) & $7(53.8 \%)$ \\
\hline Male & $6(46.2 \%)$ \\
\hline Female & \\
\hline $\begin{array}{l}\text { Creatinine, mean } \pm \text { SD } \\
\text { mg/dL }\end{array}$ & $1.0 \pm 0.3$ \\
\hline Baseline & $1.6 \pm 0.8$ \\
\hline Peak & $1.2 \pm .6$ \\
\hline Discharge & $60(57.3,64.0)$ \\
\hline Aneurysm Size, median & \\
\hline Medical Comorbidities, No. (\%) & $1(84.6 \%)$ \\
\hline Hypertension & $10(76.9 \%)$ \\
\hline Cardiac Disease & $9(69.2 \%)$ \\
\hline Hyperlipidemia & $2(15.4 \%)$ \\
\hline COPD & $3(23.5 \%)$ \\
\hline Smoker & $3(23.1 \%)$ \\
\hline Cardiomyopathy & $2(15.4 \%)$ \\
\hline CHF & $1(7.7 \%)$ \\
\hline CKD & \\
\hline History of Stroke / TIA & \\
\hline History of DVT / PE & \\
\hline Diabetes Mellitus & \\
\hline
\end{tabular}

size was $60 \mathrm{~mm}$ (IQR $57 \mathrm{~mm}-61 \mathrm{~mm}$ ). Of the preoperatively planned 30 visceral vessel targets, 29 were successfully preserved (96.7\%). No patients exhibited type I endoleak, three patients (23.1\%) demonstrated type II endoleak, and a single patient (7.7\%) demonstrated a type III endoleak within the first 30 days.

The 30-day perioperative complications and outcomes are summarized in Tables 2 and 3 respectively. Complications include one MI without need for intervention (7.7\%), one ascending aortic dissection with associated stroke (7.7\%) leading to death, one complete T8 paralysis (7.7\%) developing on postoperative day (POD) 16, a single renal artery dissection $(7.7 \%)$ leading to failed preservation, and one respiratory failure requiring tracheostomy $(7.7 \%)$. A single individual required reintervention on POD 1 for retroperitoneal hemorrhage successfully managed with coil embolization of a renal artery branch. The 30 -day mortality rate was $7.7 \%$ with a single death on POD 4 after succumbing to complications of ascending aortic arch dissection noted on POD 1. Follow up CTA obtained within one month demonstrated stable aneurysm sac size in all patients, with a median decrease in aneurysm sac size of $1.5 \mathrm{~mm}$ (IQR $0.3,3.8$ ).

\section{Discussion}

Our initial experience with parallel grafting in a high-risk, complex aneurysm patient population demonstrates high technical success with acceptable 30-day mortality and perioperative complication rates. To our surprise, there were no type I endoleaks, and low rates of type III endoleaks. Previous reports on parallel grafting also demonstrate 
Table 2. Number of patients experiencing events by category at 30 days.

\begin{tabular}{|c|c|c|}
\hline Category & No. $(\%)$ & Comments \\
\hline Death & $1(7.7 \%)$ & POD 4 Aortic Dissection \\
\hline Readmission & $5(38.5 \%)$ & \\
\hline \multicolumn{3}{|l|}{ Cardiac } \\
\hline Myocardial Infarction & $1(7.7 \%)$ & Troponin leak POD 1 - managed conservatively \\
\hline Arrhythmia & $1(7.7 \%)$ & A-fib RVR managed with rhythm control medication \\
\hline Congestive Heart Failure & $0(0 \%)$ & \\
\hline \multicolumn{3}{|l|}{ Pulmonary } \\
\hline Tracheostomy & $1(7.7 \%)$ & Inability to wean from ventilator support \\
\hline Pneumonia & $0(0 \%)$ & \\
\hline Pulmonary Embolus & $0(0 \%)$ & \\
\hline \multicolumn{3}{|l|}{ Renal } \\
\hline Acute Kidney Injury & $4(30.8 \%)$ & \\
\hline Urinary Tract Infection & $2(15.4 \%)$ & \\
\hline Dialysis & $0(0 \%)$ & \\
\hline \multicolumn{3}{|l|}{ Vascular } \\
\hline Transfusion & $3(23.1 \%)$ & \\
\hline Aortic Dissection & $1(7.7 \%)$ & POD 1 requiring aortic replacement \\
\hline Retroperitoneal Bleed & $1(7.7 \%)$ & POD 1 requiring coiling \\
\hline Renal Artery Dissection & $1(7.7 \%)$ & Left renal artery dissection with occlusion \\
\hline Perinephric Hematoma & $1(7.7 \%)$ & \\
\hline Distal Embolus & $1(7.7 \%)$ & Managed non-operatively \\
\hline Deep Venous Thrombosis & $1(7.7 \%)$ & PICC Associated Upper Extremity DVT \\
\hline Stent Occlusions & $1(7.7 \%)$ & Intra-op occlusion of left renal stent \\
\hline \multicolumn{3}{|l|}{ Gastrointestinal } \\
\hline Ischemic Colitis & $3(23.1 \%)$ & Colonoscopic evaluation of all individuals \\
\hline UGI Bleeding & $2(15.4 \%)$ & POD 1 on heparin gtt; POD 16 requiring transfusion \\
\hline Ileus & $1(7.7 \%)$ & $>4$ days managed non-operative \\
\hline Pancreatitis & $1(7.7 \%)$ & \\
\hline \multicolumn{3}{|l|}{ Neurologic } \\
\hline Stroke & $1(7.7 \%)$ & POD 1 Ischemic stroke associated with aortic dissection \\
\hline Paralysis & $1(7.7 \%)$ & POD 16 T8 complete paralysis \\
\hline \multicolumn{3}{|l|}{ Wound } \\
\hline Hematoma & $2(15.4 \%)$ & Managed without intervention \\
\hline Infection & $1(7.7 \%)$ & Groin wound infection requiring antibiotics \\
\hline Dehiscence & $0(0 \%)$ & \\
\hline
\end{tabular}

low rates of periprocedural endoleaks [13,14,16-18]. In theory, type I endoleaks are an inherent issue when molding the main body aortic graft(s) around visceral and renal snorkels. Between parallel grafts, an exposed channel exists which is susceptible to "gutter leak" around the seal zones. Increasing the length of stent overlap into healthy aorta as a means of preventing gutter leaks is debated in the literature. Some authors have suggested at least $20 \mathrm{~mm}[11,12,19,20]$ to provide an adequate seal and increased stability. Ideal stent overlap with SanEVAR repair is also unknown.

As an adjunct to overlap in the prevention of gutter leaks, aortic stent oversizing has been utilized. Acceptable oversizing percentage is debated amongst reported series $[11,14,19]$ as increased oversizing has demonstrated decreased gutter leaks but at the expense of main body endograft infolding [21]. In our series, covering a graft seal zone at least $15 \mathrm{~mm}$ into healthy aorta in primary Sn-EVAR and at least $30 \mathrm{~mm}$ between main body stents in the San-EVAR setting along with $20 \%$ oversizing appears to provide adequate seal preventing type I gutter leaks.

In addition to the debate of sizing and overlap, the utilization of balloon-expandable and self-expanding stents is also debated as both 
Table 3. Perioperative Outcomes.

\begin{tabular}{|l|l|}
\hline Perioperative & Median (IQR) \\
\hline Fluoro (min) & $64.0(56.0,91.0)$ \\
\hline Contrast (ml) & $170.0(150.0,185.0)$ \\
\hline OR Time (min) & $417.0(393.0,459.0)$ \\
\hline Surgical Time (min) & $339.0(271.0,358.0)$ \\
\hline EBL (mL) & $300.0(250.0,700.0)$ \\
\hline Baseline Cr & $1.0(0.8,1.0)$ \\
\hline Peak Cr & $1.3(1.0,2.0)$ \\
\hline Discharge Cr & $1.0(0.8,1.5)$ \\
\hline Length of Stay (days) & $8.0(6.0,10.0)$ \\
\hline Discharge Destination & No. (\%) \\
\hline Home & $10(83.3 \%)$ \\
\hline Transfer to VA (Rehab) & $1(8.3 \%)$ \\
\hline LTAC & $1(8.3 \%)$ \\
\hline Readmission & $5(41.7 \%)$ \\
\hline Follow-up & Median (IQR) \\
\hline Pre-op Aneurysm Size (mm) & $60.0(57.0,61.0)$ \\
\hline 30-day Aneurysm Decrease (mm) & $1.5(0.3,0.8)$ \\
\hline $\begin{array}{l}\text { IQR = Interquartile Range, VA = Veterans Affairs Hospital, LTAC = Long Term Acute } \\
\text { Care }\end{array}$ & \\
\hline
\end{tabular}

remain good options for target vessel preservation. Balloon expandable stents provide high radial force with increased fluoroscopic visibility allowing precise placement and fixation within the target vessel. Selfexpanding stents provide increased flexibility and kink resistance ideal for severely angulated target vessels. Previous comparisons of balloonexpandable and self-expanding stents demonstrated an increased tendency for type I endoleaks amongst self-expanding stents with no other technical differences [22]. While there remains no consensus on balloon-expandable vs. self-expanding stents, utilizing a combination of both devices and maximizing the benefits of each in approaching different target vessels is advisable. This series demonstrates that utilization of self-expanding Viabahn stents for endografting visceral vessels provides excellent technical success with no early stent related complications.

The inherent risks associated with a complex open aortic procedure must be considered in the evaluation of patients with challenging aortic anatomy. While the morbidity associated with parallel grafting is not inconsequential with rates comparable to open operations, the types of complications have changed with endovascular techniques. Wound morbidity is largely obviated with endovascular techniques, but the technical complexity of multivisceral grafting increases vascular injury including embolization, dissection, and vessel perforation. As demonstrated in our series (Table 2), accessing arch vessels lends itself to increased risk of stroke and possibly devastating aortic injury. Wire manipulation within the renal arteries leads to possible dissection and perforation. As comorbid conditions do not change in patients with degenerative aortic aneurysms, many of the postoperative complications remain similar including myocardial infarction, ischemic colitis, and acute kidney injury.

A recently published study by Katsargyris et al. [23] questioned the benefits of parallel grafting over F-EVAR and open repair based on increased rates of perioperative stroke, type I endoleak, and renal perforation in the Ch-EVAR group [23]. In contrast, Lee et al. [24] recently showed similar outcomes between parallel grafting and F-EVAR [24]. While our rate of perioperative stroke is higher than that reported by Katsargyris et al. [23], our small series size limits interpretation of its significance. A comprehensive list of complications is contained in Table 2. Unless physician modified, current F-EVAR grafts cannot treat the entire paravisceral aorta and thoracoabdominal pathologies. As Sn-EVAR and San-EVAR entail incorporation of additional visceral vessels, the level of technical complexity increases dramatically and arch vessel access may be required. As such, the rate of complications subsequently increases.

One complication not often reported within the parallel grafting literature is acute spinal cord ischemia, which one of our patient's suffered on POD 16 leading to complete T8 paralysis. At time of representation, in addition to paralysis, the patient was also found to have an active upper gastrointestinal (UGI) bleed requiring transfusion of 2 units pRBCs with esophagogastroduodenoscopy (EGD) revealing no gross hemorrhage but dusky mucosa within the body of the stomach. He had been discharged from the hospital nine days previously without concerns. It is possible that given the associated UGI bleed, acute hypotension was the inciting event leading to spinal cord ischemia. It was not possible to get an MRI at the time of presentation given the recent stent placement. He was subsequently discharged to the spinal cord rehabilitation service without further complications.

A more relevant outcome that speaks to the benefit of endovascular repair in our series is that $10(83.3 \%)$ of the 12 patients surviving to discharge returned home after a median length of stay of 8.0 (IQR 6.0, 10.0) days. The fact that a majority of patients did not immediately note significant changes in lifestyle suggests the advantages of improved quality of life following endovascular repair cannot be downplayed.

\section{Conclusion}

Endovascular treatment of complex aortic aneurysms with parallel grafting continues to evolve. Our initial experience with parallel grafting demonstrates the technique to be a viable "off-the-shelf" alternative for treating complex aneurysms with acceptable perioperative outcomes in the first 30-days.

\section{References}

1. Patel RP, Katsargyris A, Verhoeven EL, Adam DJ, Hardman JA (2013) Endovascular aortic aneurysm repair with chimney and snorkel grafts: indications, techniques and results. Cardiovasc Intervent Radiol 36: 1443-1451. [Crossref]

2. EVAR trial participants (2005) Endovascular aneurysm repair versus open repair in patients with abdominal aortic aneurysm (EVAR trial 1): randomised controlled trial. Lancet 365: 2179-2186. [Crossref]

3. Prinssen M, Verhoeven EL, Buth J, Cuypers PW, van Sambeek MR et al. (2004) A randomized trial comparing conventional and endovascular repair of abdominal aortic aneurysms. $N$ Engl J Med 351: 1607-1618. [Crossref]

4. Abbruzzese TA, Kwolek CJ, Brewster DC, Chung TK, Kang J, et al. (2008) Outcomes following endovascular abdominal aortic aneurysm repair (EVAR): an anatomic and device-specific analysis. J Vasc Surg 48: 19-28. [Crossref]

5. AbuRahma AF, Campbell J, Stone PA, Nanjundappa A, Jain A, (2009) The correlation of aortic neck length to early and late outcomes in endovascular aneurysm repair patients. J Vasc Surg 50: 738-748. [Crossref]

6. AbuRahma AF, Campbell J, Stone PA, Nanjundappa A, Scott Dean L, et al. (2010) Early and late clinical outcomes of endovascular aneurysm repair in patients with an angulated neck. Vascular 18: 93-101. [Crossref] 
7. Leurs LJ, Kievit J, Dagnelie PC, Nelemans PJ, Buth J (2006) Influence of infrarenal neck length on outcome of endovascular abdominal aortic aneurysm repair. $J$ Endovasc Ther 13: 640-648. [Crossref]

8. Greenberg RK, Sternbergh WC 3rd, Makaroun M, Ohki T, Chuter T, et al. (2009) Intermediate results of a United States multicenter trial of fenestrated endograft repair for juxtarenal abdominal aortic aneurysms. J Vasc Surg 50: 730-737. [Crossref]

9. Greenberg RK, Clair D, Srivastava S, Bhandari G, Turc A, et al. (2003) Should patients with challenging anatomy be offered endovascular aneurysm repair? J Vasc Surg 38: 990-996. [Crossref]

10. Allaqaband S, Jan MF, Bajwa T (2010) "The chimney graft"-a simple technique for endovascular repair of complex juxtarenal abdominal aortic aneurysms in no-option patients. Catheter Cardiovasc Interv 75: 1111-1115.

11. Bruen KJ, Feezor RJ, Daniels MJ, Beck AW, Lee WA (2011) Endovascular chimney technique versus open repair of juxtarenal and suprarenal aneurysms. J Vasc Surg 53: 895-904, discussion 904-5. [Crossref]

12. Coscas R, Kobeiter H, Desgranges P, Becquemin JP (2011) Technical aspects, current indications, and results of chimney grafts for juxtarenal aortic aneurysms. J Vasc Surg 53: 1520-1527. [Crossref]

13. Donas KP, Torsello G, Austermann M, Schwindt A, Troisi N, et al. (2010) Use of abdominal chimney grafts is feasible and safe: short-term results. $J$ Endovasc Ther 17 : 589-593. [Crossref]

14. Hiramoto JS, Chang CK, Reilly LM, Schneider DB, Rapp JH, et al. (2009) Outcome of renal stenting for renal artery coverage during endovascular aortic aneurysm repair. $J$ Vasc Surg 49: 1100-1106. [Crossref]

15. Ohrlander T, Sonesson B, Ivancev K, Resch T, Dias N, et al. (2008) The chimney graft: a technique for preserving or rescuing aortic branch vessels in stent-graft sealing zones. $J$ Endovasc Ther 15: 427-432. [Crossref]
16. Lee JT, Greenberg JI, Dalman RL (2012) Early experience with the snorkel technique for juxtarenal aneurysms. J Vasc Surg 55: 935-946. [Crossref]

17. Donas KP, Pecoraro F, Bisdas T, Lachat M, Torsello G, et al. (2013) CT angiography at 24 months demonstrates durability of EVAR with the use of chimney grafts for pararenal aortic pathologies. $J$ Endovasc Ther 20: 1-6. [Crossref]

18. Moulakakis KG, Mylonas SN, Avgerinos E, Papapetrou A, Kakisis JD, et al. (2012) The chimney graft technique for preserving visceral vessels during endovascular treatment of aortic pathologies. J Vasc Surg 55: 1497-1503. [Crossref]

19. Kolvenbach RR, Yoshida R, Pinter L, Zhu Y, Lin F (2011) Urgent endovascular treatment of thoraco-abdominal aneurysms using a sandwich technique and chimney grafts--a technical description. Eur J Vasc Endovasc Surg 41: 54-60. [Crossref]

20. Lachat M, Frauenfelder T, Mayer D, Pfiffner R, Veith FJ, et al. (2010) Complete endovascular renal and visceral artery revascularization and exclusion of a ruptured type IV thoracoabdominal aortic aneurysm. J Endovasc Ther 17: 216-220. [Crossref]

21. Mestres G, Uribe JP, García-Madrid C, Miret E, Alomar X, et al. (2012) The best conditions for parallel stenting during EVAR: an in vitro study. Eur J Vasc Endovasc Surg 44: 468-473. [Crossref]

22. Donas KP, Pecoraro F, Torsello G, Lachat M, Austermann M, et al. (2012) Use of covered chimney stents for pararenal aortic pathologies is safe and feasible with excellent patency and low incidence of endoleaks. J Vasc Surg 55: 659-665. [Crossref]

23. Katsargyris A, Oikonomou K, Klonaris C, Töpel I, Verhoeven EL (2013) Comparison of outcomes with open, fenestrated, and chimney graft repair of juxtarenal aneurysms: are we ready for a paradigm shift? J Endovasc Ther 20: 159-169. [Crossref]

24. Lee JT, Lee GK, Chandra V, Dalman RL (2014) Comparison of fenestrated endografts and the snorkel/chimney technique. J Vasc Surg 60: 849-856. [Crossref]

Copyright: $\odot 2015$ Kugler NW. This is an open-access article distributed under the terms of the Creative Commons Attribution License, which permits unrestricted use, distribution, and reproduction in any medium, provided the original author and source are credited. 